\title{
Trasfigurare il mondo con la fantasia. Tracce fantastiche nella narrativa breve di Elsa Morante e Anna Maria Ortese
}

\author{
Silvia ZANGRANDI ${ }^{1}$ \\ Università IULM - Milano \\ silvia.zangrandi@iulm.it
}

\begin{abstract}
RIASSUNTO
Lo studio accosta la prima produzione di Elsa Morante, rappresentata dalla narrativa breve, alla narrativa breve di Anna Maria Ortese. Diverse sono le ragioni per le quali Morante e Ortese possono essere accostate: i contenuti dei loro racconti nutrono i romanzi, i racconti giovanili di Morante possono infatti essere considerati precursori della produzione che seguirà; nella produzione di Ortese molte situazioni e diversi personaggi si riversano dalla produzione breve nei romanzi. Entrambe si caratterizzano per la loro visionarietà e i loro racconti si pongono fuori dal reale per occuparsi del reale; all'interno di storie ambientate in luoghi reali che sfumano in atmosfere senza luogo e senza tempo si svolgono le vicende da loro narrate: lo sforzo di entrambe è di avallare l'immaginario, di trovare un equilibrio tra realtà e finzione. L'affetto e la premura verso gli umili e gli indifesi, siano essi esseri umani o animali, e i riferimenti al mondo animale sono presenti sempre nei loro scritti. Entrambe sono autodidatte e amano rimarcare questa loro atipicità, alla quale si aggiungono le difficoltà che tutte le donne incontrano nell'affermarsi nel mondo. Infine, per Morante e Ortese la scrittura è considerata una pratica di conoscenza di sé e del mondo ed è l'occasione per perlustrare il proprio itinerario interiore.
\end{abstract}

Parole chiave: Morante, Ortese, racconti, visionarietà, scrittura.

\section{Transfiguring the World through Fantasy. \\ Fantastic Elements in Elsa Morante's and Anna Maria Ortese's Short Stories}

\begin{abstract}
This article compares Elsa Morante's with Anna Maria Ortese's short stories. There are several reasons why Morante and Ortese can be juxtaposed. In both cases, the content of their short stories became a significant part of their novels. Morante's early short stories can be considered as preliminary to her later output; Ortese re-used many situations and
\end{abstract}

1 Facoltà di Interpretariato, traduzione e studi linguistici e culturali; Dipartimento di Letterature Comparate e Scienze del linguaggio, via Carlo Bo 1, i-20143, Milano (Mi), Italia. 
characters of her short stories in her novels. A visionary element is evident in both authors, whose stories often take place outside common reality, even though they deal with realistic situations. Their narrations develop in real places, though filled with unrealistic details and atmospheres. They wanted to free the imagination and find an innovative balance between reality and fiction. Another common element is the love and care for humble and harmless creatures, that are numerous in their writings. Besides, both authors were self-educated, and often pointed out their atypical condition. They had the same problems to affirm their personality in a male, chauvinist milieu. For Morante and Ortese, writing was a practice of self-knowledge and a way of investigating the world.

Key words: Morante, Ortese, Short Stories, Visionariness, Writing.

«E sebbene voi dobbiate aspettarvi, o lettori, di conoscere attraverso questo libro più d'un personaggio contagiato dal nostro morbo fantastico, sappiate che il malato più grave di tutti lo avete già conosciuto. Esso non è altro se non colei che qui scrive: son io»: così Elsa Morante fa parlare Elisa in Menzogna e sortilegio. Siamo in una fase in cui Morante, lasciata alle spalle la produzione giovanile, in molti casi addirittura disconosciuta, entra nella fase più matura. Ma è noto che la sua prima produzione, rappresentata dalla narrativa breve, nutre la produzione maggiore, ovvero i romanzi ${ }^{2}$. Nel suo intervento del $1959 \mathrm{su}$ Nuovi argomenti Morante delinea la sua personale idea su romanzo e racconto: il romanzo «dà intera una propria immagine dell'universo reale» mentre il racconto rappresenta «un "momento" di realtà». Ella precisa che ciò non significa affermare la superiorità poetica del romanzo sul racconto: «non si tratta di qualità superiore o inferiore, ma di un differente rapporto con l'universo [...] Una raccolta di racconti - quando si componga $[\ldots]$ in una interezza sviluppata e armoniosa - ha valore certo di romanzo» (Morante 1959: 18-19). Grazie a questa dichiarazione è chiaro che Morante dà lo stesso valore alla produzione breve e a quella più estesa, anzi, all'interno della sua opera i racconti occupano una posizione centrale poiché precorrono e illuminano con la loro presenza l'universo stregato e straziante dei romanzi che verranno in seguito ${ }^{3}$, come vedremo tra poco.

${ }^{2}$ Sull'argomento diversi sono i contributi; mi limito a segnalarne tre. Per Marco Bardini: «è assai facile accorgersi come tali testi giovanili siano assolutamente degni di attenzione, sia per la loro evidente qualità intrinseca in rapporto al loro specifico contesto culturale, sia per la perspicuità del percorso speculativo che attraverso questi E. M. arriva a compiere» (Bardini 1999a: 462). Per Gabriella Contini: «i racconti possono anche suggerire l'ipotesi del macrotesto: come serie coerente di testi allacciati e in crescita, autonomi e interdipendenti, contrassegnati da caratteri tematico formali ricorrenti» (Contini 1993: 163). Per Giovanna Rosa: «ripercorrere la stagione dell'apprendistato "feuilletonistico" può offrire utili e insperati chiarimenti, aiutandoci a misurare la portata del mutamento e a cogliere le genesi degli elementi che ne favoriranno il pieno dispiegamento» (Rosa 1993: 58).

${ }^{3}$ Di questa opinione anche Contini 1993. 
Occuparsi di narrativa breve morantiana significa occuparsi anche di letteratura fantastica ${ }^{4}$. Nel nostro paese, diversamente dai paesi d'oltralpe, è nel XX secolo che si assiste a un'autentica fioritura di opere legate a questo "modo" letterario e la specificità del fantastico italiano va cercata proprio nel Novecento. La letteratura, libera dai vincoli di razionalismo, rappresentatività e mimeticità imposti dal positivismo, tramite il fantastico è in grado di scandagliare la psiche dell'uomo e di parlare delle ansie legate alla modernità. Il fantastico del Novecento fa leva non tanto sull'angoscia e sul terrore quanto sulla perdita di armonia con noi stessi e con il mondo attorno a noi, sul senso di smarrimento, sulla minaccia alle nostre abitudini, sull'esistenza di eventi straordinari. Il fantastico non si colloca più al di fuori della realtà, ma si àncora saldamente a fatti veri, si insinua in zone conosciute e quotidiane, fornendo della realtà immagini deformate e straordinarie, portando così l'ignoto nel noto e nel familiare. La narrazione fantastica del Novecento si nutre continuamente di elementi provenienti dal quotidiano: gli avvenimenti apparentemente straordinari e contrari alle leggi fisiche e naturali hanno luogo non in un "altro mondo", estraneo alla nostra esperienza quotidiana, ma in un mondo reale percorso abitualmente. Per dirla con Calvino, «nel Novecento è un uso intellettuale (e non più emozionale) del fantastico che s'impone: come gioco, ironia, ammicco, e anche come meditazione sugli incubi o i desideri nascosti dell'uomo contemporaneo»" (Calvino 1980 [1995: 261]). In altri termini, è necessario distinguere tra uso "emozionale" e visionario del fantastico ottocentesco e uso "intellettuale" e astratto nel Novecento. Così inteso, il fantastico può rappresentare una chiave interpretativa utile a rileggere parte della produzione narrativa di Elsa Morante.

Per avvalorare la presenza cospicua del fantastico nella narrativa novecentesca, ho pensato di accostare a Elsa Morante Anna Maria Ortese, la scrittrice novecentesca che più marcatamente ha accolto il "modo" in oggetto nel suo mondo letterario. In diverse occasioni Ortese esprime la sua ammirazione per l'opera di Elsa Morante; a proposito di L'isola di Arturo scrive: «mi colpì, e commosse, all'inizio, quell'accenno alla stella Arturo. Forse, pensavo, Elsa Morante deve aver ricordato (ricordando la stella Arturo) quel mio racconto di Angelici dolori, Il capitano, dove una ragazzetta si accorge di questo astro, e lo sceglie come confidente» (Ortese 2011: 150.) 5 . Quando ricevette nel 1988 il premio «Procida Isola di Arturo», impossibilitata a presenziare, mandò uno scritto nel quale definisce

\footnotetext{
${ }^{4}$ Secondo Garboli in Morante coesistono due registri in apparente contraddizione: «l'alto e il basso, il mitico e il qualunque, la favola e la realtà» (Garboli 1995: 25).

${ }^{5}$ Infatti, in Il capitano Ortese racconta la sua ammirazione per «alcuni astri limpidissimi [...] uno ce n'era che a tarda notte vedevo ancora, grande e solo [...] e mi pareva che costui guardasse dolorosamente turbato, bramasse chinarsi a me» (Ortese 2006: 55). Scopre trattarsi della stella Arturo, «quel tale astro verso cui pazza intimità avevo avuta un tempo» (Ivi: 60). Morante sullo stesso argomento dice: «Arturo è una stella: la luce più rapida $\mathrm{e}$ radiosa della figura di Boote, nel cielo boreale» (Morante 1957: 7).
} 
Morante «il genio più alto di tutti i tempi italiani della donna. I suoi libri sono i più grandi, tra i libri scritti da una donna italiana in qualsiasi tempo [...] belli perché sono i libri della storia del mondo»(Ortese 1993a: 43). Non è questa l'unica occasione per Ortese di parlare di Morante: «la Morante, una montagna, un genio. La critica maschile teme il genio femminile, quando è assoluto. Una donna, quando è grande scrittrice e sa andare in fondo alle cose, mette la sua mano su dolorose e mai rimarginate ferite» (Ortese 2002: XIX).

Diverse sono le ragioni grazie alle quali Morante e Ortese possono essere accostate; come vedremo, particolare attenzione verrà posta all'ambito fantastico: non solamente le nostre si caratterizzano per la loro visionarietà, ma anche per il loro impegno umano e sociale. L'affetto e la premura verso gli umili e gli indifesi, siano essi esseri umani o animali, e i riferimenti al mondo animale sono presenti sempre nella produzione di entrambe e servono per esemplificare la necessità di ricostruire l'armonia perduta. Morante scrive:

L'estrema prova di misericordia che, pur nella severità, il Padre Eterno dette all'uomo [fu di lasciargli...] la compagnia degli animali [...] grazie a loro, noi possiamo incontrare, sulla terra, uno sguardo vivente che ci dichiari l'amicizia più tenera $[\ldots]$ e chi negò che i nostri compagni animali possiedano un'anima? Costui dà prova di non possedere né cuore né criterio (Morante 1987: 19-20).

Ortese dice che è importante attuare una rivoluzione: «essa riguarda la liberazione degli altri popoli - i popoli muti di questa terra, i popoli detti Senza Anima [...] enumerare tutti i peccati dell'uomo contro il Cavallo, l'Aquila, il Passero, lo stesso Serpente [...] non si può» (Ortese 1987: 158) e poco oltre aggiunge: «io sono più che mai dalla parte delle Bestie, mi sento loro parente, o comunque un amico, un devoto» (Ortese 1987: 171).

Ortese racconta il suo amore per il mondo naturale considerato un bene comune, un soggetto e non un oggetto di possesso e sfruttamento; nei suoi scritti mette in luce il dolore per i peccati dell'umanità insensibile alla debolezza, alla differenza, alla sofferenza degli altri, dei più deboli e degli emarginati ed è convinta che «è solo l'uomo che dà il dolore, non la Bestia; e lo dà anche quando non necessario. Lo dà per gioia, per togliere qualcosa a un altro, per degradarlo» (Ortese 1987: 170). In entrambe vi è la vocazione a trovare la dimensione del sublime nei diversi, negli animali, nei sofferenti. Nel racconto Cane di Morante assistiamo all'amore e alla compassione per un cane dolce, umile, forse vile, che muore proprio per questa sua umiltà e incapacità a combattere. Il cane Lui è diverso dai soliti cani, è animato da un amore per tutti gli uomini: «rideva ogni tanto di un riso convulso, con la lingua penzolante e le orecchie basse» (Morante 2002: 102), però non sapeva azzuffarsi e questa fu la sua rovina: un aggressivo cane lupo lo azzannò e «la morte lo accompagnò fino a casa» (Morante 2002: 104). Ortese in Piccolo drago Conversazione racconta il suo dolore e la sua compassione per il "sangue animale» che ella vedeva ovunque: aveva pietà per le galline agonizzanti, per gli agnelli sgozzati, per i pesci che si dibattevano moribondi. Racconta lo strazio di un cavallo 
vecchio e macilento, tutto coperto di piaghe, che barcolla, si ferma e subisce la violenza dell'uomo che, dopo avergli sollevato la testa, gli sputa negli occhi. Commuove l'atteggiamento dell'animale che non risponde con «ira negli occhi, solo un velo, la pazienza di una vita» (Ortese 1987: 150). Ortese continua dicendo che l'uomo «sceglie i popoli e le persone (anche le Bestie sono Popoli e persone) più deboli; sceglie i non aventi diritto» (Ortese 1987: 170). Grazie agli esempi qui portati, non credo che sia arbitrario legittimare una continuità ideale tra Useppe bambino straordinario, entusiasta del mondo e di tutto ciò che lo circonda, annunciatore della presenza del divino nel mondo: «dotato di capacità superiori $\mathrm{e}$ divine, Useppe si configura come un nuovo Gesù-Cristo, un'altra volta sacrificato dalla Storia» (Martinez Garrido 2003: 91) - e Alonso di Ortese, il puma oggetto, di amore e odio, capro espiatorio che muore senza morire, la cui presenza è un monito contro le ingiustizie (Zangrandi 2008).

Morante non riusciva a concepire la sua vita senza la scrittura (lo confida a JeanNoël Schifano poco prima di morire) poiché la letteratura è da lei considerata uno strumento di interrogazione della realtà: «la poesia, come la vita, vuole proprio dare una forma e un ordine assoluti agli oggetti dell'universo, traendoli dall'informe e dal disordine» (Morante 1987: 35). Ortese, dal canto suo, è quasi certa di non essere stata uno scrittore inutile perché «oltre il mio respiro, ho appreso a desiderare il libero respiro di ogni creatura e di ogni paese» (Ortese 1997: 54): per Ortese lo scrivere è quasi un atto religioso, un modo per riscattare l'uomo dalla sua caducità e «il ribrezzo che la realtà $[\ldots]$ destava in me era sempre più grande, e sempre più intenso e disperato il desiderio di trovare scampo nella parola» (Ortese 1987: 75). Per entrambe lo scrittore deve ergersi al di sopra di ogni sistema; Morante dice «lo scrittore, per sua natura, è portato a non appartenere a nessuna società determinata, a nessun gruppo o categoria, ecc. [...] è inevitabile, comunque, che fra le classi dominanti e quelle dominate, preferisca sempre queste ultime» (Morante 1987: 114); Ortese ribadisce che gli scrittori sono «coloro che non credono, o credono poco, ai partiti, le classi, i confini, le barriere, le fazioni, le armi, le guerre. Che nel denaro non hanno posto alcuna parte dell'anima e quindi sono incomprabili. Quelli che vedono il dolore, l'abuso; vedono la bontà o l'iniquità, dovunque siano, e sentono come dovere il parlarne» (Ortese 1997: 30). Se Morante dice che alcuni trattano lo scrittore «chi da maledetto, chi da sognatore, chi da cantastorie, chi da aristocratico, chi da parente povero, chi da sovversivo, ecc. ecc.» (Morante 1987: 113), Ortese risponde che lo scrittore, «quando vorrà mostrare a che cosa, nel suo paese, e sotto gli occhi di tutti, sia ridotta la vita - discarica e ammazzatoio, dopo allevamento e oscuramento - lo si indicherà come guastatore e visionario») (Ortese 1997: 31). Se per Morante la funzione dello scrivere è «di impedire la disintegrazione della coscienza umana, nel suo quotidiano, e logorante, e alienante uso col mondo» (Morante 1987: 102), per Ortese «deve esservi un modo [...] di ricavare un insegnamento e una bellezza da tutto [...] e questo, di spiegare, illuminare, parlare, dire a tutti, sottovoce, ma eternamente, l'identità di tutti, il mistero di questo mondo, e la situazione reale di ogni popolo e di ogni uomo [...] questo potrebbe e dovrebbe essere compito di scrittori nuovi» (Ortese 1997: 46). 
E ancora, entrambe sono autodidatte e amano rimarcare questa loro atipicità, alla quale si aggiungono le difficoltà che tutte le donne incontrano nell'affermarsi nel mondo. A Ortese piaceva aggiungere alla parola scrittore la parola "donna"; Morante non voleva essere chiamata scrittrice ma scrittore e sosteneva che «è assurdo [...] dividere le scrittrici dagli scrittori: è come dividere l'umanità in biondi e bruni» (Saviane 1955: 11; cfr. anche: Andreini 2003 e Garboli 1995: 244). In un'intervista del 1960 Morante sostiene:

una donna per affermarsi con il proprio ingegno deve superare difficoltà almeno dieci volte superiori a quelle che incontrerebbe un uomo, né può mai, in definitiva, raggiungere nella società la posizione che raggiungerebbe un uomo dotato di qualità pari, o magari inferiori a quelle di lei (Morante 1988: XXVII) .

Ortese più volte mette in luce le difficoltà affrontate perché era «uno scrittoredonna, una bestia che parla», considerata come tutto il genere femminile, priva di «anima e giudizio della mente» (Ortese 1997: 51). ${ }^{6}$

I racconti di Morante e Ortese si pongono fuori dal reale per occuparsi del reale: all'interno di storie ambientate in luoghi reali che sfumano in atmosfere senza luogo e senza tempo si svolgono le vicende da loro narrate. Lo sforzo di entrambe è di avallare l'immaginario, di trovare un equilibrio tra realtà e finzione. Per Garboli, «Elsa sapeva leggere nella realtà coi sogni, le visioni, le ombre» (Garboli 1995: 195). Ortese ambiva a cogliere un'immagine e renderla «viva, grande, colorata, con tutti i caratteri precisi della realtà e tutti i deliziosi ondeggiamenti dell'irreale» (dal risvolto di Ortese 2006). L'arcano, il mistero, la «seconda realtà», il sogno che travalica, sorpassa e trasfigura il reale, le visioni che si dilatano e superano la cruda realtà sono i territori su cui si muovono le due scrittrici.

Come detto in apertura, i contenuti dei racconti sia di Morante sia di Ortese nutrono i romanzi. I racconti giovanili di Elsa Morante (Pirotti 1996; Porciani 2006) possono infatti essere considerati precursori della produzione morantiana; in essi troviamo in nuce temi, poetica, stile degli scritti futuri. Come vedremo, in Il ladro dei lumi vi è un io narrante che da adulta racconta ciò che le è accaduto quando era bambina; questo tema verrà ripreso e largamente sviluppato in Menzogna e sortilegio e in L'isola di Arturo. Un altro esempio non trascurabile è la presenza in Il ladro dei lumi di una nonna che è l'antesignana di Cesira, la nonna di Elisa di Menzogna e sortilegio. Entrambe le donne vengono rappresentate nella loro estrema decadenza: hanno la testa quasi calva, l'aspetto ossuto di uno scheletro, se ne stanno mute per ore a guardare il vuoto, sono sorde. Le fantasticherie e i sogni in cui si

${ }^{6}$ Ortese difende il connubio bestia-anima, femminilità-sapienza, e mette in campo nel suo romanzo L'Iguana, un soggetto che si presenta bestia ma che dentro di sé è un essere umano, dualismo che si esplicita come dissidio fra natura e società. Questa creatura anfibia, «stravagante e avvilita», mite e dispettosa, triste e rabbiosa, porta turbamento nel mondo e spesso soccombe alle logiche umane, soffrendo terribilmente. 
rifugiano sia Antonia in Via dell'Angelo sia i fratelli di $\mathrm{Il}_{\text {gioco }}$ segreto $^{7}$ ritorneranno in Menzogna e sortilegio quando Elisa, per sfuggire alla quotidianità triste, «richiusi i miei libri, io mi compiacevo di architettare, nella fantasia, vicende e storie di mia propria fattura, modellate, si intende, sulle mie favole predilette» (Morante 1948 [1994: 22]). E, nello stesso modo dei bambini di Il gioco segreto che giocano al teatro ${ }^{8}$ e con esso si estraniano dal mondo, Elisa dice che «alla parola teatro i miei sensi tutti all'unisono si destavano, suggerendo alla mente grande spazio, brulicare di splendori, voci strane e corali, odori di incenso. Un tumulto quasi di foresta, e una religione di cattedrali; l'esaltazione della favola, il gioco fattosi cosa divina» (Morante 1948 [1994: 496]). È quindi lecito affermare che tra $i$ racconti e i romanzi esiste un continuum. L'incantamento di Angelo nel racconto Infanzia, sospeso su una riva incantata tra acqua e terra richiama «il luogo meraviglioso» scoperto da Useppe durante le sue scorribande insieme a Bella. Angelo cresce in un luogo fiabesco: le sue madrine sono «le grandi maghe del fiume [...] dai capelli d'acqua [...] egli insegue fra balbettii rapiti il cammino del sole, e annaspa, nell'ansia di afferrare con le sue mani i frammenti di luce che danzano in giro» (Morante 2002: 156-158). Questo paesaggio fiabesco torna in La Storia: qui mancano le fate dai capelli d'acqua ma c'è sempre il fiume, il vento che muove le foglie e anche qui il paesaggio si trasfigura agli occhi di Useppe e Bella. «Erano entrati in una radura circolare, chiusa da un giro di alberi che in alto mischiavano i rami, così da trasformarla in una specie di stanza col tetto di foglie» (Morante 1974: 508). Là le fate difendono Angelo dalle vespe e lo proteggono ninnandolo con lievi canti; qui è lo spazio chiuso della radura circolare a proteggere Useppe e a stregarlo con i baluginii della luce, col movimento quieto dell'acqua e dell'aria e col cinguettio degli uccelli. Nel misterioso giovane di La via dell'angelo, come rilevato anche da Cesare Garboli, troviamo in nuce gli atteggiamenti di Carlo Vivaldi di La Storia. Il comportamento «rabbuiato», lo sguardo «sbieco e vile», gli occhi «commossi [...] le labbra si piegavano in una smorfia di delusione e di disgusto [...] pareva sbigottito dalla stanchezza» (Morante 1988: vol. I, pp. 14551457). Il camminare a stento, il pallore, i modi bruschi e scortesi del misterioso ragazzo apparso a Antonia sono i precordi dell' «abbandono disperato e indifeso [... della] faccia stravolta [... del] pallore grigio [...] la sua maniera piena di minaccia e di rancore [...] la sua fisionomia segnata da qualcosa di corrotto» (Morante 1974: 196-198) di Carlo Vivaldi.

\footnotetext{
${ }^{7}$ Attorno al «bisogno di travestire la realtà [...e] trasfigurare il mondo con la fantasia» cfr. Sgorlon 1999.

${ }^{8}$ «Nel Gioco segreto la scoperta del teatro come gioco da parte di tre fanciulli solitari e sensibili $[\ldots]$ si fa allusione alla funzione compensatoria, che la creazione artistica svolge nei confronti della vita [...] consente ai bambini, umiliati e offesi nella loro affettività, di sostituire alla realtà di solitudine e squallore, che li circonda, un mondo fiabesco d'incanti [...si tratta] della sospensione della realtà quotidiana e dell'evocazione d'una realtà parallela, affascinante [...] nella sua illusorietà visionaria. Attraverso i canali del teatro e della scrittura» (Nava 1994: 64-66).
} 
Anche nella produzione di Ortese molte situazioni e diversi personaggi si riversano dalla produzione breve nei romanzi. Il racconto Folletto a Genova per molti versi richiama i romanzi L'Iguana e Il Cardillo addolorato; è uno strano essere, come il cardillo è un po' bambino e un po' animale. Folletto-Stellino porta in testa, come l'Iguana, una pezzuola a pois, annodata in cima, come lei ha «la striminzita manina [...] simile più a un ramo di rosmarino che a una vera zampina umana» (Ortese 1987: 67); come l'Iguana sembra essere l'incarnazione del Male. Ortese, con la descrizione di figure aliene, incomprese da molti ma che una particolare sensibilità sente vive e presenti, vuole offrire mitezza da contrapporre alla crudeltà e ai dolori della vita e conclude così il racconto: «strana e potente è l'azione dell'amore (di handicappati e folletti) sull'Universo medesimo, sulla tremenda Realtà del vivere» (Ortese 1987: 172). Folletto a Genova è la triste storia di una creatura fantastica che sembra incarnare, come il puma Alonso ${ }^{9}$ dell'omonimo romanzo, un essere atto a espiare il male del mondo.

Molti racconti di Elsa Morante e di Anna Maria Ortese condividono dimensioni fantastico-surreali e, per dirla con Morante nel risvolto di Lo scialle andaluso (1963), significano «ancora una volta, la leggenda variopinta, barbara e luminosa della vita» (Bardini 1999b: 678). Nei racconti Morante mette in atto, per usare le parole di Moravia, «la psicologia in funzione dei fatti e non delle idee» (Moravia 1965: 193). Le nostre pongono alla base comportamenti alienanti, sentimenti ancestrali che si esplicitano in atmosfere surreali. Nei loro scritti le strutture che supportano la realtà come la società con $i$ suoi riti, le sue divisioni, le sue cerimonie, sono però anche la prova che la realtà non è che apparenza e quindi proprio qui vivono il mistero, il sogno, l'irrealtà. In Morante la matura sposa Elisa del racconto Il viaggio ${ }^{10}$ percorrerà come uno spirito le calli di Venezia inebriandosene; il piccolo Camillo nel racconto Innocenza ${ }^{11}$ aprirà la porta alla bella signora scarmigliata che gli ruberà la nonna; nel racconto Il figlio $^{12}$ il vecchio orologiaio vedrà nel «fanciullo con corona di cipolle» il figlio devoto che lo comporrà nella bara; il cocchiere ${ }^{13} \mathrm{e}$ il padrone correranno in una tempesta nera per tutta l'eternità, senza mai raggiungere la meta. In tutti risalta, per dirla con Garboli, il bisogno di

${ }^{9}$ Alonso «è fratello di altri tre miei racconti, anzi quattro per altro brevissimi [...] questi racconti erano: Sulla terrazza sterminata, Di notte e Bambini della creazione e buon ultimo Folletto a Genova» (Secchieri 2005: 1122).

${ }^{10} \mathrm{Il}$ racconto Il viaggio uscì in I diritti della scuola, numero del 20 settembre 1938. Ora si legge in Morante (2002).

${ }^{11}$ Il racconto Innocenza uscì in Oggi, numero del 25 novembre 1939. Ora si legge in Morante (2002).

${ }^{12}$ Il racconto Il figlio uscì in I diritti della scuola, numero del 2-9 aprile 1939. Ora si legge in Morante (2002).

${ }^{13}$ Il racconto Il cocchiere uscì in Oggi, numero dell'11 novembre 1939, apparve poi in Morante (1941) e non venne più ripubblicato successivamente. Ora si legge in Morante (2002). 
stregare la realtà e la capacità di trasfigurarla con «un lampo visionario». Ortese dissemina elementi fiabeschi in molti suoi racconti: in Isola compare una località «strana, dolce e selvaggia» (Ortese 2006: 13) nella quale la protagonista non sente più né fame né freddo né stanchezza. Nel Pellerossa l'eroe Cavallo Bianco appare grande e luminoso col capo circondato da «un'aureola di penne» (Ortese 2006: 24) e campeggia gigantesco come una divinità sulla parete della camera dei ragazzi e li osserva con sguardo «grave, sdegnoso, dolorante» (Ortese 2006: 25).

Morante e Ortese condividono un uso particolare del tempo e dello spazio che crea un'atmosfera surreale e inquietante che permea tutti i loro scritti. Lo spazio e il tempo sfuggono alla norma: lo spazio a volte si restringe a una via, a vicoli bui, a una finestra, a una cappella, a volte si dilata in vastità immerse nella nebbia. Il tempo a volte è racchiuso in precisazioni cronologiche, a volte invece c'è confusione tra passato e presente in una sospensione temporale tra realtà e sogno. Del resto in Menzogna e sortilegio Morante dice che il fluire metodico del tempo è parvenza:

il passato e il futuro, infatti, sono due campi di nebbia e di vertigine, che i vivi non possono esplorare se non con la fantasia e con la memoria; ma forse fantasia e memoria sono soltanto strumenti d'illusione, e soltanto per un gioco ingannevole l'uomo crede di avere il passato alle spalle e il futuro innanzi a sé. In realtà egli si muove sopra una sfera immobile (Morante 1948 [1994: 233]).

L'illusorietà del tempo viene ribadita in Aracoeli: «il tempo - che gli uomini tentano di domare con gli orologi, fino a renderlo un automa - è per se stesso di natura vaga, imprevedibile e multiforme» (Morante 1988: vol. II, p. 1273). La via dell'Angelo è un racconto pervaso da atmosfere surreali e fantastiche create dalla leggenda dell'Angelo di pietra che si trova sull'incrocio di una via; di lui si narrava che si introducesse nelle case sotto varie forme per rapire la gente, specialmente i bambini. Tutto l'ambiente è immerso in un alone misterioso, a Antonia le strade incutono molta paura e a ogni piccolo rumore trasale pensando che sia l'angelo che la vuol rapire. Lo spazio misterioso circonda la ragazzina con immagini surreali: dai vetri ella vede «una valle deserta piena di un lontano e misterioso chiarore [...] inaccessibili alture, a picco una casa solitaria, ricca di torri e contrafforti, allungata da altissime guglie» (Morante 1988: vol. I, p. 1458) ${ }^{14}$. Questa immagine fiabesca allevia, seppur per poco, l'atmosfera lugubre delle strade buie e strette, della casa col soffitto basso, il pavimento di mattoni sconnessi e la parete chiazzata di umidità. La topografia e la qualità delle cose serve a evidenziare una realtà ordinata che però viene a scontrarsi con uno spazio indefinibile dove bellezza e corruzione si mescolano e si confondono. Se in Via dell'Angelo Antonia si perdeva ad ammirare gli angeli musicanti sulle vetrate e godeva dei colori che si proiettavano sul soffitto e le pareva di volare in una nube colorata, in Il gioco segreto i tre fratelli, attraverso

${ }^{14}$ Il racconto La via dell'Angelo uscì in Meridiano di Roma, 14 agosto 1938; poi in Morante 1941; poi in Morante 1963; infine in Morante 1988. 
una recita teatrale, si estraniano dal vissuto quotidiano triste e noioso per entrare in un mondo fiabesco fatto di dame, cavalieri, cacciatori con i cani, cavalli e suonatori di corno. «Così viva era la forza della finzione, che ciascuno dimenticava la propria persona reale» (Morante 1988: vol. I, p. 1469) ${ }^{15}$. Questo gioco si svolgeva in un pianeta favoloso e lontano e spesso i tre fanciulli non dormivano la notte per pensare alla recita e metterla in atto. Una notte intorno a loro tutto si materializza: «Antonietta era in piedi presso un albero dipinto nel quale d'improvviso cominciò a scorrere la linfa» (Morante 1988: vol. I, p. 1472). La ragazzina si ritrova indosso una veste lunga, di forma sontuosa; il fratello Giovanni è diventato un «gagliardo cavaliere» e Pietro un suonatore di corno. Ma questa finzione, nella quale i tre si rifugiavano per compensare i divieti e le meschinità che li circondavano, è una chimera che viene bruscamente interrotta dall'arrivo dei genitori: «in quel momento [...] gli alberi e i cavalieri si irrigidirono, senza più dimensioni, e un silenzio polveroso entrò nella stanza. Alla luce delle candele non c'erano che tre brutti fanciulli» (Morante 1988: vol. I, p. 1474). Questo improvviso ritorno alla realtà si conclude con il triste rientro alla vita di tutti i giorni dove Giovanni, dopo aver tentato la fuga, cade nella malattia; Antonietta gli sta accanto «simile a una serva di convento» (Morante 1988: vol. I, p. 1478). Alle brillanti luci della recita dove ci sono dame dalle «vesti fantastiche adorne di gemme, e reti intessute d'oro» (Morante 1988: vol. I, p. 1467) fa da contraltare la casa patrizia in rovina, i putti corrosi e sudici, il portale macchiato di muffa, i fiori azzurrastri e patiti del giardino. Il mondo della fantasticheria, dell'illusione, che Morante chiamerà anche «menzogna» è labile ed effimero, il fascino del gioco segreto scompare e la vita nel palazzo fatiscente e squallido si svolgerà come in una prigione. Anche Anna Maria Ortese in Il capitano (Ortese 2006: 54-65) racconta la complicità con il fratello Antonio fatta di invenzioni e fantasia; ai due si aggiunge Franco, il fratello più piccolo. Insieme trasformano la terrazza, che si trova al piano superiore della loro casa, in uno spiazzo enorme e straordinario dove passano le giornate, consumano alcuni pasti, osservano il cielo e le stelle, si scambiano racconti e riflessioni. Ma, come in Il gioco segreto, anche nel racconto di Ortese la pace, l'immersione nel fantastico, la gioia nel trovarsi soli di fronte al creato, il piacere nell'immaginare di vivere indicibili avventure nella tenda innalzata sulla terrazza vengono inaspettatamente interrotte dalle grida dei parenti che distruggono con violenza la tenda-casa e annullano nei bambini il piacere di vivere fiabesche avventure.

Ortese ci offre spesso ambientazioni straordinarie e perturbanti: il racconto Sulla terrazza sterminata si apre con una casa dove accadevano strani fatti; segue la descrizione dettagliata dell'appartamento dove misteriosi e inquietanti fenomeni suggeriscono «l'eterna sensazione di una terza presenza invisibile» (Ortese 1987: 140). Strani e sinistri accadimenti vengono descritti dall'inquilina della casa: la nascita di «un'aurora gigantesca di un colore rosa molto vicino al ciclamino»; i

15 Il racconto Il gioco segreto uscì in Meridiano di Roma, 13 giugno 1937; poi in Morante 1941; poi in Morante 1963; infine in Morante 1988. 
«piccoli orologi di legno colorato [...] che da anni non funzionavano più, ma in quella casa ripresero tutti, disordinatamente e quasi ininterrottamente a suonare le ore»; le «chiavi di casa che ora si trovavano sul pavimento di una stanza [...] facevi per raccoglierle, ti sfuggivano [...] l'improvviso e inspiegabile avvicinarsi di due oggetti simili» (Ortese 1987: 141; 143); e infine la scomparsa delle sigarette da un pacchetto lasciato sul tavolo. Questi eventi inspiegabili sono smagliature del mondo sensibile che ci circonda e che mostrano la fragilità della realtà fatta di causa ed effetto, dietro alla quale ognuno di noi si barrica per non restare disorientato. Spesso poi in Ortese sogno fa rima con allucinazione. La casa del bosco è un racconto attraversato da uno stato allucinatorio difficile da descrivere e, come ella stessa dice, «l'ACCADUTO (alquanto indefinibile) [...] forma l'argomento di questa storia» (Ortese 1987: 16). Una lunghissima e precisissima descrizione della casa e dei suoi dintorni fa da cornice al racconto vero e proprio: ci viene detto il numero delle porte, delle finestre, il loro orientamento, la lunghezza e la larghezza del cortile, la narratrice giunge perfino a dirci il numero delle piastrelle che rivestono il muro. La narratrice attende un idraulico per sistemare il tubo della cucina che perde acqua e allaga la casa; il tempo di attesa è pervaso da terrore e angoscia: il vento intona «un canto inarticolato, selvaggio, ora simile a lamento, ora a minaccia, ora a una lunga storia di vittime» (Ortese 1987: 20). Il terrore è tale che induce la protagonista a sprangare porte e finestre e infine, stanca dell'attesa, si addormenta e sogna. Inizia qui la descrizione di immagini inconsistenti, inspiegabili, confuse e illogiche del sogno Inesplicabilmente la narratrice continua anche dopo il risveglio a stabilire stretti legami tra sogno e realtà: ciò che ha immaginato di vedere e ciò che realmente ha visto si mescola in modo misterioso. Le interferenze dell'inconscio qui descritte in un insieme di informazioni confuse, di desideri, di reminiscenze, di paure, di domande emergono nel sonno e nel sogno e da esso vengono proiettate nel reale: Ortese ha rappresentato attraverso il sogno l'insieme delle informazioni molteplici e confuse in cui è immerso il soggetto umano.

Con frequenza sia nei racconti di Morante sia in quelli di Ortese appaiono figure di revenant. Nel racconto Il ladro dei lumi di Morante compaiono fantasmi silenziosi usciti dalle mura del tempio: sono le ombre dei morti rimasti senza un lume a rischiarare la tenebra dove sono immersi perché il guardiano Jusvin, anziché alimentare le lampade con l'olio, ne intascava il tributo ${ }^{16}$. I fantasmi vengono descritti in maniera tale da atterrire la bambina che li incontra: sono masse oscure con il volto dalle occhiaie vuote. Tutto il racconto è immerso in un'atmosfera inquietante: il buio diventa un fantasma che mostra «al posto degli occhi due fosse

${ }^{16}$ In una conversazione con Jean-Noël Schifano, Morante informa che «è un racconto. Non è una favola: è una storia vera. A Modena c'era un ebreo che era molto, molto povero, allora andava a rubare l'olio dei lumi dei morti, e mia nonna lo vedeva dalla finestra e me lo ha raccontato... no, non mia nonna, non l'ho mai conosciuta. Ma quel personaggio è vero» (Schifano 1993: 10). 
nere» (Morante 1988: vol. I, p. 1410) ${ }^{17}$; Jusvin lascia dietro di sé un buio enorme dopo aver spento le lampade. Anche il tempo, la cui cronologia è confusa, conferisce ambiguità alla narrazione: 1'incipit mostra una ragazzina di circa sei anni che però alla fine, mescolando presente e passato, sostiene: «quella ragazzina fui io, o forse mia madre, o forse la madre di mia madre; io sono morta e rinata, e ad ogni nascita si inizia un nuovo processo incerto» (Morante 1988: vol. I, p. 1414). Nel racconto La nonna Morante sfrutta il sentimento di gelosia e possesso di un figlio da parte di una madre portandolo al massimo del parossismo e dell'assurdo. La nuora, appena la vede, sente «il guizzare di uno sguardo nero» (Morante 1988: vol. I, p. 1429$)^{18}$ : tramite questa sinestesia il racconto si incammina verso il perturbante. La nonna, dopo la nascita dei due bimbi, se ne va da casa e dopo la sua partenza «le stanze apparivano fresche e deserte» (Morante 1988: vol. I, p. 1439): ma questa donna lugubre e crudele tornerà per breve tempo, il tempo di raccontare ai due bambini una fiaba. Rivediamo qui gli elementi fiabeschi a cui Morante si era dedicata negli anni trenta, scrivendo per il Corriere dei piccoli. Mi sostiene in questa opinione Claude Cazalé: «nella fiaba troviamo in nuce temi e motivi che compariranno, ora capovolti ora parodiati, ora ironicamente e nostalgicamente metaforizzati, nei racconti e romanzi»»(Cazalé Bérard 2009: 184). Dopo essersi suicidata gettandosi nel torrente, comparirà appoggiata alla parete di fronte al letto dei due coniugi, volgendo alla nuora Elena i suoi occhi ammiccanti. Anche il figlio vede il fantasma di sua madre e si sveglia angosciato. Saranno i capelli di Giuseppe, improvvisamente incanutiti, a fare da oggetto mediatore tra il mondo reale e la visita notturna della terribile nonna. Il fantasma di questa donna è di una crudeltà indescrivibile perché, in maniera perturbante sotto forma di farfalla nera e rossa, attirerà i bambini sulla riva del torrente facendoli annegare. Il lettore si trova immerso in un'atmosfera ambigua, tra una realtà compatta e palpabile presentata con cura maniacale e una realtà autre, estranea a quella parvente che perturba e spaventa.

Legato a un'ambientazione ancora ottocentesca è il racconto Il cocchiere dove il giovane studente che narra la storia entra nella camera del vecchio zio giudice attratto da soffi, fischi e rumori strani e sospetti e vede il giudice, «un vecchio di statura gigantesca e piuttosto pingue, con le guance cascanti e pallide» (Morante 2002: 15). Costui però dice di essere il cocchiere e di avere ucciso il suo padrone giudice; spiega che la violenza è stata perpetrata come reazione al suo licenziamento e precisa: «siamo dannati tutti e due [...] cocchiere e padrone in eterno corriamo sulla carrozza, per una tempesta nera, e non arriveremo mai» (Morante 2002: 16) e aggiunge che i parenti non sanno che lui è morto. Improvvisamente la camera è invasa da una bufera di vento e le ciocche bianche del

${ }^{17}$ Il racconto Il ladro dei lumi porta la data del 1935 ma uscì per la prima volta in Morante 1963.

${ }^{18}$ Il racconto La nonna uscì in Meridiano di Roma, 18 agosto 1937; poi in Morante 1941; poi in Morante 1963. 
vecchio si agitano furiose, i suoi denti battono, la sua pelle illividisce.

Di fantasmi si occupa anche il racconto L'uomo degli occhiali, ma si tratta di fantasmi che poco hanno a che vedere con quelli terrificanti prima narrati. Anche qui siamo immersi in una dimensione fantastica e assurda ${ }^{19}$ benché la realtà circostante sia normale: la portinaia saluta il protagonista, la lattaia gli prepara la colazione, il paesaggio è innevato. Il tempo però ha preso una frustata: 1'uomo dagli occhiali è convinto che sia domenica, invece è giovedì e non sa che cosa sia successo in questi giorni. Davanti alla scuola, dove aspettava una bambina, gli viene detto che costei è morta. Quest'uomo goffo, con le spalle ricurve, scompare nella nebbia e a questo punto il racconto prende una svolta assolutamente fantastica. Clara, l'amica di Maria che è morta, incontra la ragazzina in un luogo stranissimo dove «un popolo oscuro si aggirava con una velocità febbrile [...] e di questa folla senza numero ella non riusciva a distinguere le facce né la foggia dei vestiti» (Morante 1988: vol. I, p. 1419) ${ }^{20}$. Il fantasma di Maria non ha nulla di orrifico, il suo viso era smagrito e aveva molte rughe, le sue pupille erano opache e la sua faccia era invecchiata, "con gli occhi asciutti che parevano ingranditi da un bistro» (Morante 1988: vol. I, p. 1422). Clara circondata da questo spazio illimitato e sconosciuto non è intimorita, prende per mano l'amica e infine insieme ridono piano.

Similmente si muove Anna Maria Ortese: il singolare racconto Il Fantasma narra di morti che ritornano e si svolge in un'atmosfera strana ma che non spaventa. Qui una narratrice senza nome, a distanza di anni, racconta, confessando però di aver forse sofferto «un'allucinazione» (Ortese 2001: 69) ${ }^{21}$, l'incontro con lo zio Alberto, morto da giovane ma improvvisamente riapparso da uomo maturo. Tuttavia l'uomo che accompagna Alberto, Enrico, è il vero protagonista; si tratta di un'entità identificabile con la morte, ma chiamata nel corso della narrazione anche «il fantasma» (Ortese 2001: 96). Da un lato viene descritto «l'orribile scheletro» sormontato «da un piccolo teschio dall'aria intelligente e mordace» da cui spunta una lingua nera e occhi di tenebra, indossa un orologio «a forma di falce» (Ortese 2001: 81) e i suoi aiutanti sono «tre alte e bianche figure [...] avvolte in un lungo sudario bianco, forato in alto da due buchi, da cui trasparivano gli occhi degli spettri, stranamente verdi e fiammanti» (Ortese 2001: 110). Dall'altro però certe dichiarazioni del fantasma («amo la musica», Ortese 2001: 100), certi atteggiamenti («singhiozzava [...] smarrito», Ortese 2001: 114) servono a indicare la perdita

\footnotetext{
${ }^{19}$ Per questo racconto Morante confessa la sua ammirazione per Kafka. Anche Angelo R. Pupino (Pupino 1968) mette in luce il debito verso Kafka, distinguendo però tra l'originalità della prosa morantiana per niente asservita a quella dello scrittore praghese. Inoltre, se l'angoscia e l'incubo ricordano Kafka, qui l'assurdo non diventa una vera realtà, ma si mescola con le zone buie dell'inconscio.

${ }^{20}$ Il racconto L'uomo degli occhiali uscì in Meridiano di Roma, 25 aprile 1937; poi in Morante 1941 e infine in Morante 1963.

${ }^{21}$ Il racconto Il fantasma apparve a puntate tra il 1941 e il 1942 in otto numeri della rivista Nove Maggio; ora in Ortese 2001: 63-129.
} 
dell'aspetto orrifico e consentono a questo scritto di rispecchiare la generale attitudine novecentesca nei confronti del fantastico. Gli elementi più destabilizzanti si riferiscono all'atteggiamento della morte, impersonata da Enrico, che soffre di solitudine e si sente «così solo, così infelice, così noncurato e spesso temuto, così desideroso di vivi affetti [...] Quanto vorrei essere amato, signor mio! E non mi riesce» (Ortese 2001: 118).

Prima di concludere, vorrei mettere a confronto due racconti che si occupano di opere d'arte che si animano ${ }^{22}$. I due racconti si offrono come esempio di trasgressione del confine tra realtà e rappresentazione e cancellano i limiti tra percezione sensoriale e visione onirica (Mangini 2007: 225). Secondo Lugnani si tratta di un tema legato alla produzione dei capolavori della pittura e della scultura «che devono potersi muovere e parlare perché sono così perfetti da parere vivi» (Lugnani 1983: 209). Nella scelta dei racconti qui fatta, l'ekphrasis è posta su dipinti e sculture che ancora una volta alludono alla possibilità di una vita alternativa a quella reale, ma anche alla duplicità dell'essere, al ritorno del rimosso, alla frattura tra coscienza e inconscio. Attorno a un volto di donna dipinto sulla parete di un palazzo veneziano che bisbiglia chissà quale segreto si sviluppa il racconto Il viaggio di Elsa Morante. Nel racconto la visita di due sposi alla città di Venezia è il pretesto per descrivere in maniera fiabesca la città fatta d'acqua e popolata d'angeli. Morante riesce magistralmente a recuperare il passato illustre e senza tempo della città lagunare: la sua bellezza urbanistica e l'atmosfera che unicamente lì si respira trapelano nel solo nominare i termini campielli, calli, rii, canali $^{23}$. La protagonista del racconto di Morante vive la sua alterità geografica in quanto turista nella città di Venezia e mostra continuamente la sua non appartenenza ai luoghi dove si trova (prima della partenza è smarrita e si sente «venir meno»; durante il viaggio è «stupefatta e intimorita»). Il viaggio autentico è preceduto da quello onirico: «Venezia legava una sensazione di giubilo soffocato, mista a uno smarrimento [...]. Le pareva di vedere Venezia in forma di mare tranquillo, su cui enormi angeli di marmo camminavano senza toccar l'acqua, coi piedi nudi, le lunghe vesti scendenti» (Morante 2002: 126). Giunti a Venezia, si mantiene, anche grazie alle scelte linguistiche, questa sensazione di irrealtà: le case riflettono i loro fantasmi nell'acqua immobile, la piazza sterminata e deserta sembra un lago pieno di favolosi riflessi, la chiesa è un luccichio di gemme, santi e colori, la città intera «pareva dissolversi in fantasmi liquidi e oscillanti» (Morante 2002: 129). A questo esordio impalpabile segue la visita a un meraviglioso palazzo dove Elisa vede un

${ }^{22}$ Per una disamina dell'argomento cfr. Pellini 2001.

23 Nello stesso anno in cui pubblica Il viaggio, Morante scrive un articolo per Prospettive; in esso si domanda: «esiste davvero per sé una realtà concreta del mondo, o di esso non vi sono che infinite apparenze [...]? O forse ancora il vero aspetto della realtà è inscrutabile, nessuno può vederla quale essa è, ma ciascuno porta in sé un'apparenza del mondo, limitata alla sua propria sensibilità?». (Bardini 1999a: 465-466). L'articolo, dal titolo «Mille città in una», apparve in Prospettive, 4-5, (1938). 
quadro raffigurante una donna bellissima. Tramite immagini eteree racchiuse nella bocca dall'ambiguo sorriso, la dama del dipinto pare voglia svelare a Elisa un segreto. Da qui in poi si è immersi nella levità del sogno e di un mondo irreale: circondata dalla presenza costante dell'acqua, elemento sfuggente per antonomasia, dai balconi scolpiti e trapunti, dai ricami d'oro, dai marmi istoriati, la protagonista ha l'impressione di essere «uno spirito, un'ombra» portata in stato di sonno in questo mondo irreale. Il linguaggio accumula immagini che si intrecciano, si raddoppiano, svaniscono, per ricomparire in un susseguirsi di pensieri, sensazioni e avvenimenti il cui apice è dato dal ritratto della donna-fanciulla che con grazia solleva il piede nudo sotto la veste e con la bocca piegata in un ambiguo sorriso teso a bisbigliare chissà quale segreto, turba la donna a tal punto da farla sentire simile $\mathrm{a}$ uno spirito ${ }^{24}$.

Anna Maria Ortese, invece, tratta il tema della statua che si anima nel racconto L'infanta sepolta; la «piccola Maestà Nera» ortesiana incarna l'immagine della devozione popolare e l'evento perturbante descritto da Ortese mostra la capacità di cogliere anche nelle cose più banali aspetti alieni, incompresi dalla maggioranza, ma percepiti da chi possiede una sensibilità diversa. Ortese rileva il lato cupo e oscuro della vita dell'Infanta, mette in scena l'indistinto e l'irrazionale attraverso un muto linguaggio di gesti; pare che la statua si animi per comunicare alla narratrice la sua vita di dolori. La statua dell'Infanta segnala alla narratrice la sua condizione inspiegabile e crudele di prigioniera: «può darsi che tutte queste non fossero che fantasticherie, torbide supposizioni di un cuore che, ieri come oggi, è portato a vedere dovunque dei prigionieri, a riconoscere in ogni albero un carcere di spiriti ardenti, in ogni sasso una cella infame, dove qualcuno arde e si lamenta» (Ortese 1948; 1950 [2000: 68] $)^{25}$. Diversamente dai quadri e dalle statue narrate dagli scrittori ottocenteschi, che vogliono spaventare e sono spesso causa di sventura, Morante e Ortese con questi due racconti ci riferiscono eventi da considerare, per dirla con Ortese, «un improvviso indebolirsi della ragione»(Ortese 1948; 1950 [2000: 68]) che percepisce quanto sta al di là della soglia del reale.

In conclusione, per Morante e Ortese la scrittura è considerata una pratica di conoscenza di sé e del mondo ed è l'occasione per perlustrare il proprio itinerario interiore. Obiettivo comune è di suscitare nell'uomo una nuova coscienza per ricavarne un insegnamento e una bellezza. La quotidianità si trasfigura in un mondo che supera il reale e le vicende oniriche alimentano i loro scritti ma non rappresentano un'uscita dalla realtà, semmai un approfondimento della stessa, un aspetto intrinseco alla realtà. Nei loro racconti mettono in campo il bene e il male, la menzogna e la verità, la finzione e la realtà. Elsa Morante sembra dirci che la

\footnotetext{
${ }^{24}$ Il confronto tra questo racconto e altri due a firma di Dino Buzzati e Giorgio Vigolo è stato oggetto di un mio studio dal titolo Città reali oltre il reale: incursioni del fantastico a Milano, Venezia, Roma (Zangrandi 2012).

${ }^{25}$ L'infanta sepolta uscì in Risorgimento, 8 aprile 1948, p. 3; poi in Ortese 1950 [2000].
} 
realtà non è che apparenza e proprio in essa vivono il mistero e il sogno. Dal canto suo Ortese è convinta che sia difficile «trattare del reale! In parte perché nessuna cosa, a pensarci, è veramente reale [...] L'invenzione, al contrario, è lieta e rassicurante» (Ortese 1993b: 21).

\section{RIFERIMENTI BIBLIOGRAFICI}

ANDREINI, Alba (2003): La Morante e il diario: autoritratto di donna e di scrittrice, in Martínez Garrido, Elisa y cols. (ed), Elsa Morante. La voce di una scrittrice e di un'intellettuale rivolta al secolo XXI, Madrid, Departamento de Filología Italiana de la Universidad Complutense de Madrid, pp. 9-22.

BARDINI, Marco (1999a): «Scheda sugli esordi editoriali di Elsa Morante», in Italianistica, XXVIII, 3, pp. 461-467.

BARDINI, Marco (1999b): Morante Elsa. Italiana. Di professione, poeta, Pisa, Nistri-Lischi, Appendice I.

CALVINO, Italo (1980 [1995]): Una pietra sopra, Einaudi, Torino.

CAZAlÉ BÉRARD, Claude (2009): Donne tra memoria e scrittura. Fuller, Weil, Sachs, Morante, Roma, Carocci.

CONTINI, Gabriella (1993): «Elsa Morante: autoritratti d'autrice. Dal Meridiano di Roma allo Scialle andaluso», in Annali della Facoltà di Lettere e Filosofia dell'Università di Siena, XIV, pp. 163-175.

GARBOLI, Cesare (1995): Il gioco segreto. Nove immagini di Elsa Morante, Milano, Adelphi.

Lugnani, Lucio (1983): Per una delimitazione del "genere", in Ceserani, Remo et al. (a cura di), La narrazione fantastica, Pisa, Nistri-Lischi.

MANGINI, Angelo (2007): Letteratura come anamorfosi. Teoria e prassi del fantastico nell'Italia del primo Novecento, Bologna, Bononia University Press.

MARTINEZ GARRIDO, Elisa (2003): Bestiario, allegoria e parola ne La Storia di Elsa Morante. Un'altra vita verso il sacro, in Martínez Garrido, Elisa y cols. (ed), Elsa Morante. La voce di una scrittrice e di un'intellettuale rivolta al secolo XXI, Madrid, Departamento de Filología Italiana de la Universidad Complutense de Madrid, pp. 85-107.

MORANTE, Elsa (1941): Il gioco segreto, Garzanti, Milano

MORANTE, Elsa (1948 [1994]): Menzogna e sortilegio, Torino, Einaudi.

MORANTE, Elsa (1957): L'isola di Arturo, Torino, Einaudi.

MORANTE, Elsa (1959): «Nove domande sul romanzo», Nuovi argomenti, 38-39, pp. 17-38.

MORANTE, Elsa (1963): Lo scialle andaluso, Torino, Einaudi.

MORANTE, Elsa (1974): La Storia, Torino, Einaudi.

MORANTE, Elsa (1987): Pro o contro la bomba atomica e altri scritti, a cura di Cesare Garboli, Milano, Adelphi. 
MoRANTE, Elsa (1988): Opere, a cura di Carlo Cecchi e Cesare Garboli, 2 voll., Milano, Mondadori.

MORANTE, Elsa (2002): Racconti dimenticati, a cura di Irene Babbioni e Carlo Cecchi, Torino, Einaudi.

MORAVIA, Alberto (1965): Racconto e romanzo in L'uomo come fine, Milano, Bompiani.

NAVA, Giuseppe (1994): «Il Gioco segreto di Elsa Morante: i modi del racconto», Studi novecenteschi, 47-48, XXI, pp. 53-78.

ORTESE, Anna Maria (1948; 1950 [2000]): L'infanta sepolta, Milano, Adelphi.

ORTESE, Anna Maria (1987): In sonno e in veglia, Milano, Adelphi.

ORTESE, Anna Maria (1993a): Il nudo respiro potente di secoli perduti in Notarbartolo, Schifano (ed.), Cahiers Elsa Morante, Napoli, Edizioni Scientifiche Italiane, pp. 43-44.

ORTESE, Anna Maria (1993b): «Così canto il Cardillo», in Corriere della Sera, (30 maggio 1993), p. 21.

ORTESE, Anna Maria (1997): Corpo celeste, Milano, Adelphi.

ORTESE, Anna Maria (2001): Il Monaciello di Napoli, Milano, Adelphi.

ORTESE, Anna Maria (2002): Romanzi, vol. I, a cura di Monica Farnetti, Milano, Adelphi.

ORTESE, Anna Maria (2006): Angelici dolori e altri racconti, a cura di Luca Clerici, Milano, Adelphi.

ORTESE, Anna Maria (2011): Da Moby Dick all'Orsa Bianca. Scritti sulla letteratura e sull'arte, a cura di Monica Farnetti, Milano, Adelphi.

PELLINI, Pierluigi (2001): «Il tema del quadro animato nella letteratura del secondo Ottocento», Belfagor, LVI, 1, pp. 11- 33.

PirotTI, Umberto (1996): «Sulle opere giovanili d'Elsa Morante», in Studi e problemi di critica testuale, LIII, 53, pp. 159-184.

PORCIANI, Elena (2006): L'alibi del sogno nella scrittura giovanile di Elsa Morante, Soveria Mannelli, Iride.

PUPINO, Angelo R. (1968): Strutture e stile della narrativa di Elsa Morante, Ravenna, Longo.

RosA, Giovanna (1993): Ovvero: il romanziere, in Agamben, Giorgio (ed.), Per Elsa Morante, Milano, Linea d'ombra edizioni, pp. 55-87.

SAVIANE, Sergio (1955): «Elsa Morante e L'isola di Arturo», L'Espresso, 2 ottobre 1955.

SCHIFANO, Jean-Noël (1993): La divina barbara, in Tjuna Notarbartolo / Jean-Noël Schifano (ed.), Cahiers Elsa Morante, Napoli, Edizioni Scientifiche Italiane, pp. $5-13$.

SECCHIERI, Filippo (2005): Alonso e $i$ visionari. Note ai testi in Ortese, Anna Maria (ed.): Romanzi, vol. II, Milano, Adelphi.

SGORLON, Carlo (1999): «Elsa Morante tra realtà e fantasia», Nuova Antologia, 134, pp. 196-208.

ZANGRANDI, Silvia (2008): «Dare voce alle cose del mondo prive di voce. Alonso e i visionari di Anna Maria Ortese», Italianistica, XXXVII, 2, pp.141-148. 
ZANGRANDI, Silvia (2012): Città reali oltre il reale: incursioni del fantastico a Milano, Venezia, Roma, in Barenghi / Langella / Turchetta (ed.): La città e l'esperienza del moderno, tomo III, Pisa, Edizioni ETS, pp. 443-452. 